\title{
Selected Haematological and Biochemical Indices of Nile Tilapia (Oreochromis niloticus) Reared in the Environment with Cyanobacterial Water Bloom
}

\author{
Miroslava Palíková1, Radovan Kopp ${ }^{2}$, Jan Mareš², Stanislav Navrátili1, Zdenek Kubíček³, \\ Lubomír Chmelař ${ }^{4}$, Hana Band'ouchová1, Jiří Pikula ${ }^{1}$ \\ ${ }^{1}$ Department of Veterinary Ecology and Environmental Protection, University of Veterinary and Pharmaceutical \\ Sciences Brno, Czech Republic \\ ${ }^{2}$ Department of Fishery and Hydrobiology, Mendel University of Agriculture and Forestry, Brno, \\ Czech Republic \\ ${ }^{3}$ Department of Clinical Biochemistry, Hospital Trinec, Czech Republic \\ ${ }^{4}$ Medistat, Brno, Czech Republic \\ Received September 18, 2009 \\ Accepted April 6, 2010
}

\begin{abstract}
The aim of this study was to evaluate the influence of toxic cyanobacterial water blooms on blood indices in the Nile tilapia (Oreochromis niloticus). Experimental fish were exposed to natural cyanobacterial water blooms (consisting mainly of Microcystis aeruginosa and M. ichthyoblabe) which contained microcystins (total concentration $1187-1211 \mu \mathrm{g}^{-\mathrm{g}^{-1}}$ of dry weight and 17.4 $25.4 \mu \mathrm{g} \cdot \mathrm{l}^{-1}$ of water) for 28 days without additional feeding. Control groups of fish were kept in another pond without apparent cyanobacterial bloom formation. Experimental and control rearing ponds had the same water source. After exposure, fish were placed in dechlorinated potable water for the same period.

Statistical evaluation of the influence of cyanobacterial water bloom on biochemical indices of experimental fish showed a distinct increase of alkaline phosphatase $(p \leq 0.05)$, total bilirubin $(p \leq 0.001)$, creatinine $(p \leq 0.01)$, lactate $(p \leq 0.01)$ and urea $(p \leq 0.01)$ when compared to controls. After transfer to the dechlorinated potable water the experimental group showed significantly lower values of phosphorus $(p \leq 0.001)$, urea $(p \leq 0.01)$ and cholinesterase $(p \leq 0.05)$ and higher values of lactate $(p \leq 0.05)$ and iron $(p \leq 0.05)$ compared to controls. It may be concluded that the exposure of the Nile tilapia to the environment containing cyanobacterial water bloom influenced only some biochemical indices. However, this modulation is to a much lower degree compared to the common carp and silver carp.
\end{abstract}

Microcystins, fish, haematology, biochemistry, blue-green algae

Eutrophication of freshwater bodies due to increased exogenous nutrient loading and the consequent massive development of water bloom represents a problem throughout the world. It is known that cyanobacterial secondary metabolites, i.e. cyanotoxins, cause a wide range of health disorders in animals as well as humans.

The most widespread cyanobacterium in freshwater ecosystems of Europe, Microcystis aeruginosa together with others (Microcystis sp., Oscillatoria sp., Anabaena sp., Nostoc sp.) can produce microcystins (MCs) (Malbrouck and Kestemont 2006). Hepatotoxic MCs, synthesized during the algal growth phase, are released from damaged cells when the bloom is collapsing (Ros s et al. 2006). Cyanobacteria and fish live in the same environment so there may be close interactions. Fish can be exposed to cyanotoxins via food intake. It has been suggested that phytoplanktonophagous fish species may be more affected by cyanobacterial toxins due to higher digestion of the cyanobacterial water bloom (Carbis et al. 1997; Vajcova et al. 1998). On the other hand, experiments with the silver carp proved its strong resistance to toxic Microcystis bloom and MCs (Shen et al. 2005). Another route of exposure to cyanotoxins is through the skin or gills (Malbrouck and Kestemont 2006), but it is considered to be negligible (Tencalla et al. 1994).

Some authors report the influence of experimentally administered MCs on haematological and plasma chemistry indices (Rabergh et al. 1991; Vajcova et al. 1998; Navratil et al.

Address for correspondence:

MVDr. Miroslava Palíková, Ph.D.

Department of Veterinary Ecology and Environmental Protection

University of Veterinary and Pharmaceutical Science Brno

Palackeho 1-3, 61242 Brno, Czech Republic
Phone: +420 541562821 ,

Fax: +420 541562654

e-mail: palikovam@vfu.cz

http://www.vfu.cz/acta-vet/actavet.htm 
1998; Li et al. 2004) in different fish species. Intraperitoneal exposure to microcystins or lysates of cyanobacterial biomass applied orally cause significant changes of biochemical indices, red blood cells and activities of plasma enzymes. MCs cause liver tissue damage in fish demonstrated by the significant increase in alanine aminotransferase (ALT), aspartate aminotransferase (AST) and lactate dehydrogenase (LDH) (Rabergh et al. 1991; Vaj cova et al. 1998; Navratil et al. 1998).

The rate of increase of LDH, ALT and AST depends on the route of administration, characteristics of the material, and the amount of toxin. Fish exposure to media containing dispersed MCs demonstrated that toxic effects are time-delayed. The toxic effect after oral administration is approximately 10 times weaker than after the intraperitoneal administration (Carbis et al. 1997).

It is difficult to observe the long-term impact of cyanobacteria containing microcystins at lower concentrations in individual fish. Therefore, it is more useful to evaluate more individuals in a fish population. The measurement of aminotransferases (ALT, AST), bile acids, bilirubin, sodium and chloride from the blood serum is recommended (Carbis et al. 1996). Biochemical indices of blood in fish may be affected by many endogenous and exogenous factors. The changes of haematological and biochemical indices in fish could be caused either by MCs or other chemical factors (Kopp and Hetesa 2000; Luskova et al. 2002; Pepeljnjak et al. 2003), nutrition (Serpunin 1995) and stress (Dobsikova et al. 2006).

In tilapia fish, microcystins induce oxidative stress in a time-dependent manner under laboratory conditions (Jos et al. 2005; Prieto et al. 2006; 2007). Atencio et al. (2009) and Prieto et al. (2008) investigated the role of selenium and vitamin E supplementation, respectively, on the oxidative stress and histopathological changes induced by cyanobacterial cells containing microcystins in tilapia fish. The effects of low and repeated doses of microcystin LR from cyanobacterial cells on the enzymatic activities of ACP and ALP were investigated also underlaboratory conditions by Molina et al. (2005). ACP and ALP activities changed in a time-dependent manner and these changes were more evident in liver and kidney. No influence on the biochemical indices was noted.

All the above mentioned studies with tilapia fish were conducted under laboratory conditions. The aim of this work was to study in natural conditions the effects of a natural cyanobacterial population with known amounts of microcystins on biochemical indices of the Nile tilapia (Oreochromis niloticus).

\section{Materials and Methods}

\section{Experimental fish}

Nile tilapia (Oreochromis niloticus) specimens obtained from a single artificial stripping (Fishpond Tisova, Czech Republic) were used for the experiment. These fish measured $211 \pm 14 \mathrm{~mm}$ in length, and had an average body weight $171 \pm 34 \mathrm{~g}$. The fish specimens were acclimatised for 1 week before the start of the study in a small pond without cyanobacteria. They were caged and exposed (100 fish) to cyanobacterial bloom which naturally developed in the breeding pond for 28 days during the months of August to October, 2006. In parallel, control group (100 fish) of fish were also kept in another pond without apparent cyanobacterial bloom formation. Fish were reared under natural conditions without additional feeding. After exposure, they were placed in pure water for the same period of time (i.e., 28 days) in two 1000-1 tanks containing dechlorinated drinking water. Fish were exposed to a $12 \mathrm{~h} \mathrm{light} / 12 \mathrm{~h}$ dark photoperiod, and the tank water was changed daily. Properties of water in breeding ponds (given for experimental and control group, respectively) were as follows: water temperature 17.8 $\pm 1.3,17.9 \pm 1.3^{\circ} \mathrm{C}$; dissolved oxygen $93 \pm 32,114 \pm 35 \%$; $\mathrm{pH} .2 \pm 0.6,8.5 \pm 0.8$, ammonia $0.22 \pm 0.06,0.18$ $\pm 0.12 \mathrm{mg} \cdot \cdot^{-1} \mathrm{~N}-\mathrm{NH}_{4}$. Properties of pure water were as follows: water temperature $26.7 \pm 0.2$; dissolved oxygen $60 \pm 10 \% ; \mathrm{pH} 7.3 \pm 0.2$; ammonia $2.0 \pm 1.5 \mathrm{mg} \cdot \mathrm{l}^{-1} \mathrm{~N}-\mathrm{NH}_{4}$. Water saturation by oxygen, temperature and $\mathrm{pH}$ were measured by a WTW Oxi 340i dissolved oxygen meter and a WTW pH 340i pH meter (WTWGmbH, Germany). Ammonium ions were determined by the Nessler method and nitrites using the N-(1-naphthyl)-ethylenediamine method (APHA 1981).

Phytoplankton and microcystins

Cyanobacterial and algal biomass was evaluated every week by total chlorophyll $a$ concentrations (ISO 1992) 
and by the number of cells counted in the Bürker's counting chamber. Cyanobacterial biomass (dominated by coccal Microcystis aeruginosa and M. ichthyoblabe) estimated by the chlorophyll $a$ cell concentration varied from 65 to $206 \mu \mathrm{g} \cdot \mathrm{l}^{-1}\left(169-971 \times 10^{3}\right.$ cells in $\left.1 \mathrm{ml}\right)$ in the experimental pond. The algal biomass (dominated by chlorococcal green algae), also estimated by the chlorophyll $a$ cell concentration, varied from 5 to $203 \mu \mathrm{g} \cdot \cdot^{-1}$ (1.5$107 \times 10^{3}$ cells in $1 \mathrm{ml}$ ) in the control pond.

Concentrations of total microcystins in the cyanobacterial and algal biomass were determined by a previously published method using HPLC (Agilent 1100 system, Supelcosil ABZ+Plus C18 column; Agilent Technologies Inc., Santa Clara, CA, USA) coupled with a photodiode array detector (Blaha and Marsalek 2003). The concentrations of microcystins in the experimental pond were $1187-1211 \mu \mathrm{g} \cdot \mathrm{g}^{-1}$ of DW biomass and $17.4-25.4$ $\mu \mathrm{g} \cdot \mathrm{l}^{-1}$ of water. The concentrations are rather comparable with microcystin levels from other ponds in the Czech Republic (Marsalek et al. 2001).

Haematological and biochemical indices

Sampling of fish was conducted at the start of the experiment and then on an every-seventh-day basis during and after the exposure. Immediately after catching the fish (10 experimental and 10 control ones in every weak) from a pond or tank, blood samples were collected. Fish blood was taken by cardiopuncture using heparinised syringes. Heparin at a concentration of 50 I.U. per $1 \mathrm{ml}$ was used for blood stabilization. Inadequate and haemolytic specimens of blood were eliminated. Values of haemoglobin ( $\mathrm{Hb}$ ), haematocrit (PCV), mean corpuscular haemoglobin concentration (MCHC), mean corpuscular volume (MCV), mean corpuscular haemoglobin (MCH), erythrocyte (RBC) and leukocyte (WBC) counts were determined by standard methods (Svobodova et al. 1991).

The blood was centrifuged at $400 \mathrm{~g}$ for $15 \mathrm{~min}$ at $4{ }^{\circ} \mathrm{C}$ and the resulting plasma stored at $-80{ }^{\circ} \mathrm{C}$ until the day of analyses. Biochemical analyses of blood plasma were conducted using commercially available reagents using the ADVIA 1650 automatic analyzer (Bayer- Tarrytown, USA). All serum enzymatic activities were analyzed at $37^{\circ} \mathrm{C}$.

Statistical analyses

Significance of the difference between every individual experimental and control groups was analysed by $t$-test using the Unistat 5.0 software. Significantly different indices compared to the control are marked by one asterisk $(P<0.05)$ or two asterisks $(P<0.01)$.

\section{Results}

Tables 1 to 4 present the main results of this study. In Table 1 there are results of haematological indices of fish exposed to cyanobacterial biomass in experimental ponds and in Table 2 there are results of haematological indices of fish transferred into dechlorinated potable water without any cyanobacteria. There were no significant changes of values compared to controls. In Table 3 there are results of biochemical indices of fish exposed to cyanobacterial biomass in experimental ponds. Statistical analysis of these data

Table 1. Haematological indices of fish in experimental ponds, i.e. first 28 days, weeks $1-4$ (mean $\pm \mathrm{SD}, \mathrm{n}=10$ )

\begin{tabular}{|l|l|c|c|c|c|}
\hline Week & 1 & 2 & 3 & 4 \\
\hline \multirow{2}{*}{$\begin{array}{l}\text { Erythrocyte } \\
\text { number }\left(.10^{12} \cdot \mathrm{l}^{-1}\right)\end{array}$} & exposed fish & $1.85 \pm 0.24$ & $2.01 \pm 0.26$ & $1.92 \pm 0.24$ & $1.81 \pm 0.35$ \\
\cline { 2 - 6 } & control fish & $1.78 \pm 0.25$ & $2.00 \pm 0.39$ & $1.87 \pm 0.18$ & $2.04 \pm 0.19$ \\
\hline \multirow{2}{*}{ Haemoglobin $\left(\mathrm{g} \cdot \mathrm{l}^{-1}\right)$} & exposed fish & $85.0 \pm 8.5$ & $71.4 \pm 12.4$ & $74.3 \pm 4.6$ & $73.1 \pm 5.1$ \\
\cline { 2 - 6 } & control fish & $84.2 \pm 10.1$ & $74.5 \pm 11.3$ & $70.9 \pm 6.9$ & $77.5 \pm 5.5$ \\
\hline \multirow{2}{*}{ Haematocrit $\left(1 \cdot 1^{-1}\right)$} & exposed fish & $0.30 \pm 0.03$ & $0.28 \pm 0.04$ & $0.29 \pm 0.02$ & $0.27 \pm 0.02$ \\
\cline { 2 - 6 } & control fish & $0.30 \pm 0.04$ & $0.28 \pm 0.03$ & $0.27 \pm 0.02$ & $0.29 \pm 0.02$ \\
\hline \multirow{2}{*}{ MCH $\left(.10^{-12} \mathrm{~g}\right)$} & exposed fish & $46.4 \pm 5.9$ & $36.0 \pm 5.9$ & $39.3 \pm 4.5$ & $41.5 \pm 6.4$ \\
\cline { 2 - 6 } & control fish & $47.7 \pm 5.6$ & $37.6 \pm 3.8$ & $38.1 \pm 4.7$ & $38.4 \pm 4.2$ \\
\hline \multirow{2}{*}{ MCV $\left(.10^{-15} \mathrm{l}\right)$} & exposed fish & $166.0 \pm 20.0$ & $137.7 \pm 19.1$ & $151.5 \pm 15.4$ & $153.2 \pm 26.4$ \\
\cline { 2 - 6 } & control fish & $167.0 \pm 17.9$ & $142.3 \pm 17.7$ & $144.8 \pm 11.8$ & $144.9 \pm 9.3$ \\
\hline \multirow{2}{*}{ MCHC $\left(1 \cdot 1^{-1}\right)$} & exposed fish & $0.28 \pm 0.01$ & $0.26 \pm 0.02$ & $0.26 \pm 0.01$ & $0.27 \pm 0.02$ \\
\cline { 2 - 6 } & control fish & $0.29 \pm 0.02$ & $0.27 \pm 0.02$ & $0.26 \pm 0.01$ & $0.26 \pm 0.01$ \\
\hline \multirow{2}{*}{$\begin{array}{l}\text { Leukocyte } \\
\text { count }\left(.10^{9} \cdot 1^{-1}\right)\end{array}$} & exposed fish & $79.5 \pm 16.9$ & $93.4 \pm 28.0$ & $77.9 \pm 18.5$ & $100.9 \pm 32.0$ \\
\cline { 2 - 6 } & control fish & $70.1 \pm 19.3$ & $75.6 \pm 21.9$ & $71.0 \pm 30.2$ & $92.5 \pm 18.2$ \\
\hline
\end{tabular}

Abbreviations: mean corpuscular haemoglobin concentration $(\mathrm{MCHC})$, mean corpuscular volume (MCV), mean corpuscular haemoglobin $(\mathrm{MCH})$ 
Table 2. Haematological indices of fish in pure water, i.e. the second 28 days, weeks 5-8 (mean $\pm \mathrm{SD}, \mathrm{n}=10)$

\begin{tabular}{|c|c|c|c|c|c|}
\hline \multicolumn{2}{|l|}{ Week } & 5 & 6 & 7 & 8 \\
\hline \multirow{2}{*}{$\begin{array}{l}\text { Erythrocyte } \\
\text { number }\left(10^{12} \cdot 1^{-1}\right)\end{array}$} & exposed fish & $1.68 \pm 0.17$ & $1.72 \pm 0.21$ & $1.88 \pm 0.23$ & $1.91 \pm 0.15$ \\
\hline & control fish & $1.59 \pm 0.19$ & $1.77 \pm 0.20$ & $1.82 \pm 0.32$ & $1.92 \pm 0.27$ \\
\hline \multirow{2}{*}{ Haemoglobin $\left(\mathrm{g} \cdot \mathrm{l}^{-1}\right)$} & exposed fish & $84.5 \pm 7.2$ & $75.4 \pm 4.8$ & $75.9 \pm 8.5$ & $101.9 \pm 5.6$ \\
\hline & control fish & $80.5 \pm 7.7$ & $70.3 \pm 5.9$ & $71.9 \pm 6.9$ & $98.9 \pm 5.5$ \\
\hline \multirow{2}{*}{ Haematocrit $\left(1 \cdot 1^{-1}\right)$} & exposed fish & $0.26 \pm 0.02$ & $0.27 \pm 0.02$ & $0.27 \pm 0.03$ & $0.27 \pm 0.02$ \\
\hline & control fish & $0.25 \pm 0.02$ & $0.26 \pm 0.03$ & $0.27 \pm 0.03$ & $0.27 \pm 0.02$ \\
\hline \multirow{2}{*}{$\mathrm{MCH}\left(\cdot 10^{-12} \mathrm{~g}\right)$} & exposed fish & $50.8 \pm 5.9$ & $44.4 \pm 5.4$ & $40.7 \pm 4.9$ & $53.6 \pm 4.7$ \\
\hline & control fish & $51.0 \pm 5.6$ & $40.0 \pm 4.5$ & $40.0 \pm 4.1$ & $52.2 \pm 6.3$ \\
\hline \multirow{2}{*}{$\operatorname{MCV}\left(\cdot 10^{-15} 1\right)$} & exposed fish & $158.3 \pm 18.1$ & $156.6 \pm 19.4$ & $145.8 \pm 16.5$ & $140.0 \pm 13.8$ \\
\hline & control fish & $157.5 \pm 23.1$ & $145.6 \pm 15.8$ & $148.1 \pm 20.0$ & $140.7 \pm 14.1$ \\
\hline \multirow{2}{*}{$\operatorname{MCHC}\left(1 \cdot 1^{-1}\right)$} & exposed fish & $0.32 \pm 0.02$ & $0.28 \pm 0.02$ & $0.28 \pm 0.01$ & $0.38 \pm 0.02$ \\
\hline & control fish & $0.33 \pm 0.04$ & $0.28 \pm 0.03$ & $0.27 \pm 0.02$ & $0.37 \pm 0.02$ \\
\hline \multirow{2}{*}{$\begin{array}{l}\text { Leukocyte count } \\
\left(\cdot 10^{9} \cdot 1^{-1}\right)\end{array}$} & exposed fish & $69.8 \pm 17.1$ & $56.6 \pm 15.5$ & $73.5 \pm 32.6$ & $84.1 \pm 19.3$ \\
\hline & control fish & $60.9 \pm 10.7$ & $78.9 \pm 30.0$ & $70.1 \pm 33.5$ & $85.0 \pm 24.8$ \\
\hline
\end{tabular}

Abbreviations: mean corpuscular haemoglobin concentration (MCHC), mean corpuscular volume (MCV), mean corpuscular haemoglobin $(\mathrm{MCH})$

revealed an increase of ALP, total bilirubin, creatinine, urea, cholesterol and a decrease and a successive increase of $\mathrm{Mg}$ of exposed fish compared to the control fish. In Table 4 there are results of biochemical indices of fish transferred into dechlorinated potable water without any cyanobacteria. Statistical analysis of the data revealed an increase of lactate and iron and a decrease of total protein, urea, cholinesterase and phosphorus of exposed fish in comparisson to control.

\section{Discussion}

This study was focused on the effects of a natural cyanobacterial population with known amounts of microcystins on haematological and biochemical indices of the Nile tilapia (Oreochromis niloticus). Values of haematological and biochemical indices in this work only partially correspond with the results published by other authors (Tencalla et al. 1994; Navratil et al. 1998; Rabergh et al. 1991; Carbis et al. 1996; Kopp and Hetesa 2000). Statistical analysis of the data revealed an increase of ALP, total bilirubin (day 7), creatinine (day 28), urea (day 14), cholesterol (day 21) and a decrease (day 14) and a successive increase (day 21) of $\mathrm{Mg}$ in fish exposed to cyanobacterial biomass and an increase of lactate and iron (day 35) and a decrease of total protein (day 56), urea (day 35), cholinesterase (day 42) and phosphorus (day 56) in fish transferred into dechlorinated potable water without any cyanobacteria. Liver enzymes (ALT, AST and LDH) are the most frequently tested enzymes for the indication of cyanobacterial toxicity in fish. Although our results showed an increase, no significant changes of these indices of hepatic damage were found. On the other hand, we found an increased value of total bilirubin which may indicate hepatic injury. The influence of toxic substances increased the values of bilirubin in the common carp (Pepeljnjak et al. 2003). The concentration of bilirubin rises $8 \mathrm{~h}$ after intraperitoneal injection of microcystins (Carbis et al. 1996). Higher concentration of toxic cyanobacteria in a natural lake caused the increase of bilirubin concentration in the serum of feral carps (Carbis et al. 1997).

Compared to the control fish, exposure to cyanobacteria had effects on the serum ALP and cholinesterase (CHE) activities. An increased value of alkaline phosphatase indicates incorrect secretion of bile. The decrease of cholinesterase values may indicate chronic injury of the hepatopancreas. It has been shown that serum activities of ALP significantly 
Table 3. Plasmatic indices of fish in experimental ponds, i.e. first 28 days, weeks 1-4 (mean $\pm S D, n=10$ ).

\begin{tabular}{|c|c|c|c|c|c|}
\hline \multicolumn{2}{|l|}{ Week } & 1 & 2 & 3 & 4 \\
\hline \multirow{2}{*}{$\begin{array}{l}\text { ACP } \\
\left(\mu \text { kat } \cdot l^{-1}\right)\end{array}$} & exposed fish & $0.18 \pm 0.09$ & $0.10 \pm 0.04$ & $0.10 \pm 0.03$ & $0.16 \pm 0.05$ \\
\hline & control fish & $0.15 \pm 0.09$ & $0.07 \pm 0.02$ & $0.18 \pm 0.24$ & $0.21 \pm 0.23$ \\
\hline \multirow{2}{*}{$\operatorname{ALB}\left(\mu \mathrm{kat} \cdot \mathrm{l}^{-1}\right)$} & exposed fish & $7.63 \pm 1.76$ & $7.37 \pm 1.15$ & $6.51 \pm 0.94$ & $8.86 \pm 1.07$ \\
\hline & control fish & $6.69 \pm 3.13$ & $7.27 \pm 1.47$ & $7.20 \pm 1.03$ & $8.17 \pm 1.23$ \\
\hline \multirow{2}{*}{$\operatorname{ALP}\left(\mu \mathrm{kat} \cdot \cdot^{-1}\right)$} & exposed fish & $0.63 \pm 0.28^{*}$ & $0.44 \pm 0.17$ & $0.45 \pm 0.22$ & $0.58 \pm 0.24$ \\
\hline & control fish & $0.32 \pm 0.21$ & $0.46 \pm 0.23$ & $0.43 \pm 0.19$ & $0.53 \pm 0.17$ \\
\hline \multirow{2}{*}{$\operatorname{ALT} \quad\left(\mu \mathrm{kat} \cdot \mathrm{l}^{-1}\right)$} & exposed fish & $4.97 \pm 4.65$ & $0.87 \pm 0.97$ & $1.30 \pm 0.76$ & $3.31 \pm 1.79$ \\
\hline & control fish & $1.20 \pm 1.27$ & $0.72 \pm 0.43$ & $0.88 \pm 0.83$ & $2.22 \pm 2.06$ \\
\hline \multirow{2}{*}{$\operatorname{BIL}\left(\mu \mathrm{mol} \cdot 1^{-1}\right)$} & exposed fish & $5.96 \pm 2.27 * *$ & $6.11 \pm 2.44$ & $5.71 \pm 3.93$ & $8.06 \pm 2.76$ \\
\hline & control fish & $0.44 \pm 0.80$ & $6.06 \pm 2.50$ & $7.67 \pm 4.25$ & $5.47 \pm 2.11$ \\
\hline \multirow{2}{*}{ CRE $\left(\mu \mathrm{kat}^{-1^{-1}}\right)$} & exposed fish & $17.0 \pm 5.8$ & $11.4 \pm 2.3$ & $14.1 \pm 2.3$ & $18.5 \pm 2.1$ \\
\hline & control fish & $17.7 \pm 2.7$ & $10.5 \pm 2.2$ & $11.8 \pm 2.6$ & $13.2 \pm 3.1$ \\
\hline \multirow{2}{*}{$\operatorname{AST}\left(\mu \mathrm{kat} \cdot \mathrm{l}^{-1}\right)$} & exposed fish & $10.22 \pm 6.44$ & $3.15 \pm 2.97$ & $6.49 \pm 4.51$ & $9.34 \pm 4.50$ \\
\hline & control fish & $4.55 \pm 3.82$ & $3.06 \pm 1.45$ & $3.00 \pm 2.25$ & $5.89 \pm 6.23$ \\
\hline \multirow{2}{*}{$\mathrm{Ca}\left(\mathrm{mmol} \cdot \cdot^{-1}\right)$} & exposed fish & $4.73 \pm 1.31$ & $4.52 \pm 0.97$ & $4.49 \pm 1.34$ & $5.29 \pm 1.66$ \\
\hline & control fish & $4.97 \pm 1.71$ & $4.92 \pm 0.99$ & $5.09 \pm 1.24$ & $5.43 \pm 1.37$ \\
\hline \multirow{2}{*}{$\mathrm{TP}\left(\mathrm{g} \cdot \mathrm{l}^{-1}\right)$} & exposed fish & $32.5 \pm 6.9$ & $33.9 \pm 4.8$ & $32.9 \pm 3.9$ & $40.4 \pm 3.3$ \\
\hline & control fish & $30.6 \pm 9.5$ & $32.6 \pm 3.9$ & $32.4 \pm 2.8$ & $31.2 \pm 11.5$ \\
\hline \multirow{2}{*}{$\operatorname{GLC}\left(\mathrm{mmol} \cdot \mathrm{l}^{-1}\right)$} & exposed fish & $6.13 \pm 3.89$ & $3.20 \pm 1.08$ & $2.42 \pm 0.53$ & $4.70 \pm 2.04$ \\
\hline & control fish & $6.88 \pm 4.53$ & $2.69 \pm 0.56$ & $2.92 \pm 1.62$ & $3.44 \pm 0.68$ \\
\hline \multirow{2}{*}{$\operatorname{Mg}\left(\mathrm{mmol} \cdot \cdot^{-1}\right)$} & exposed fish & $1.40 \pm 0.21$ & $0.88 \pm 0.12^{* * * *}$ & $1.04 \pm 0.13^{* * *}$ & $0.99 \pm 0.16$ \\
\hline & control fish & $1.19 \pm 0.34$ & $1.05 \pm 0.09$ & $0.79 \pm 0.12$ & $0.88 \pm 0.13$ \\
\hline \multirow{2}{*}{ LACT $\left(\mathrm{mmol} \cdot 1^{-1}\right)$} & exposed fish & $1.02 \pm 0.32$ & $0.71 \pm 0.29$ & $1.14 \pm 0.35$ & $1.25 \pm 0.51$ \\
\hline & control fish & $1.04 \pm 1.11$ & $0.82 \pm 0.31$ & $0.57 \pm 0.21$ & $1.01 \pm 0.42$ \\
\hline \multirow{2}{*}{$\mathrm{LDH}\left(\mu \mathrm{kat} \cdot \mathrm{l}^{-1}\right)$} & exposed fish & $37.1 \pm 18.2$ & $22.3 \pm 22.7$ & $33.8 \pm 24.3$ & $44.5 \pm 25.0$ \\
\hline & control fish & $24.8 \pm 23.9$ & $18.0 \pm 10.6$ & $14.7 \pm 11.7$ & $25.9 \pm 26.9$ \\
\hline \multirow{2}{*}{$\mathrm{P}\left(\mathrm{mmol} \cdot \mathrm{l}^{-1}\right)$} & exposed fish & $4.43 \pm 0.70$ & $2.72 \pm 0.51$ & $3.22 \pm 0.50$ & $3.86 \pm 0.61$ \\
\hline & control fish & $3.69 \pm 1.17$ & $3.15 \pm 0.29$ & $3.13 \pm 0.47$ & $3.38 \pm 0.34$ \\
\hline \multirow{2}{*}{$\mathrm{Fe}\left(\mu \mathrm{mol} \cdot 1^{-1}\right)$} & exposed fish & $25.9 \pm 10.9$ & $18.9 \pm 5.4$ & $23.9 \pm 8.5$ & $24.9 \pm 5.5$ \\
\hline & control fish & $21.6 \pm 10.4$ & $21.6 \pm 4.9$ & $21.5 \pm 5.4$ & $23.4 \pm 2.4$ \\
\hline \multirow{2}{*}{$\mathrm{U}\left(\mathrm{mol} \cdot \cdot^{-1}\right)$} & exposed fish & $0.63 \pm 0.31$ & $0.38 \pm 0.09$ *** & $0.32 \pm 0.17$ & $0.48 \pm 0.18$ \\
\hline & control fish & $0.52 \pm 0.25$ & $0.19 \pm 0.13$ & $0.29 \pm 0.13$ & $0.37 \pm 0.19$ \\
\hline \multirow{2}{*}{$\operatorname{CHE}\left(\mu \mathrm{kat} \cdot \mathrm{l}^{-1}\right)$} & exposed fish & $7.54 \pm 3.75$ & $9.18 \pm 2.93$ & $8.77 \pm 1.99$ & $9.26 \pm 4.32$ \\
\hline & control fish & $6.81 \pm 3.06$ & $7.77 \pm 2.52$ & $10.94 \pm 5.84$ & $8.99 \pm 5.05$ \\
\hline \multirow{2}{*}{$\mathrm{CHOL}\left(\mathrm{mmol} \cdot 1^{-1}\right)$} & exposed fish & $5.40 \pm 2.04$ & $7.95 \pm 2.68$ & $6.89 \pm 2.82^{* * *}$ & $6.53 \pm 1.20$ \\
\hline & control fish & $6.19 \pm 3.49$ & $5.87 \pm 2.20$ & $5.38 \pm 1.22$ & $7.10 \pm 0.73$ \\
\hline
\end{tabular}

Abbreviations: acid phosphatase (ACP), albumin (ALB), alkaline phosphatase (ALP), alanine aminotransferase (ALT), total bilirubin (BIL), creatinine (CRE), aspartate aminotransferase (AST), calcium (Ca), total protein (TP), glucose (GLC), magnesium (Mg), lactate (LACT), lactate dehydrogenase (LDH), phosphorus (P), iron $(\mathrm{Fe})$, urea $(\mathrm{U})$, cholinesterase (CHE), cholesterol (CHOL), * $(p<0.05), * *(p<0.01)$.

decreased and activities of CHE significantly increased in the silver carp exposed to toxic cyanobacterial population (Kopp et al. 2005). We noted increased ALP and total bilirubin at the same time (day 7) and decreased CHE activities after the transfer of fish into dechlorinated potable water without any cyanobacteria (day 42). Our resulting activities of alkaline phosphatase and cholinesterase support the assumption that the liver tissue of experimental fish was slightly affected. Likewise, the increased values of creatinine (day 28), observed in the experimental fish group, indicate that the toxic population of 
Table 4. Plasmatic indices of fish in pure water, i.e. the second 28 days, weeks 5-8 (mean $\pm \mathrm{SD}, \mathrm{n}=10)$.

\begin{tabular}{|c|c|c|c|c|c|}
\hline \multicolumn{2}{|l|}{ Week } & 5 & 6 & 7 & 8 \\
\hline \multirow{2}{*}{$\operatorname{ACP}\left(\mu\right.$ kat $\left.^{-1} \mathrm{l}^{-1}\right)$} & exposed fish & $0.20 \pm 0.11$ & $0.12 \pm 0.05$ & $0.11 \pm 0.05$ & $0.12 \pm 0.05$ \\
\hline & control fish & $0.11 \pm 0.05$ & $0.13 \pm 0.06$ & $0.09 \pm 0.03$ & $0.13 \pm 0.05$ \\
\hline \multirow{2}{*}{$\operatorname{ALB}\left(\mu \mathrm{kat} \cdot \cdot^{-1}\right)$} & exposed fish & $9.19 \pm 1.57$ & $9.39 \pm 2.01$ & $9.81 \pm 1.65$ & $8.37 \pm 0.97$ \\
\hline & control fish & $9.23 \pm 0.97$ & $9.73 \pm 0.90$ & $9.86 \pm 2.07$ & $9.51 \pm 1.83$ \\
\hline \multirow{2}{*}{$\operatorname{ALP}\left(\mu \mathrm{kat} \cdot 1^{-1}\right)$} & exposed fish & $0.59 \pm 0.25$ & $0.72 \pm 0.27$ & $0.75 \pm 0.24$ & $0.59 \pm 0.17$ \\
\hline & control fish & $0.58 \pm 0.21$ & $0.67 \pm 0.16$ & $0.72 \pm 0.11$ & $0.59 \pm 0.11$ \\
\hline \multirow{2}{*}{$\operatorname{ALT}\left(\mu \mathrm{kat} \cdot 1^{-1}\right)$} & exposed fish & $1.10 \pm 1.65$ & $1.44 \pm 1.27$ & $0.81 \pm 1.02$ & $0.37 \pm 0.12$ \\
\hline & control fish & $0.87 \pm 0.58$ & $1.44 \pm 1.52$ & $0.35 \pm 0.08$ & $0.90 \pm 1.03$ \\
\hline \multirow{2}{*}{$\operatorname{BIL}\left(\mu \mathrm{mol} \cdot 1^{-1}\right)$} & exposed fish & $0.04 \pm 0.11$ & $0.44 \pm 0.46$ & $0.23 \pm 0.56$ & $0.00 \pm 0.00$ \\
\hline & control fish & $0.20 \pm 0.33$ & $0.21 \pm 0.27$ & $0.16 \pm 0.34$ & $0.04 \pm 0.11$ \\
\hline \multirow{2}{*}{$\operatorname{CRE}\left(\mu \mathrm{kat} \cdot \mathrm{l}^{-1}\right)$} & exposed fish & $20.8 \pm 3.9$ & $25.7 \pm 9.0$ & $25.5 \pm 6.2$ & $29.1 \pm 9.3$ \\
\hline & control fish & $25.0 \pm 9.1$ & $34.1 \pm 10.5$ & $32.2 \pm 21.2$ & $39.4 \pm 20.3$ \\
\hline \multirow{2}{*}{$\operatorname{AST}\left(\mu \mathrm{kat} \cdot 1^{-1}\right)$} & exposed fish & $3.40 \pm 3.56$ & $4.13 \pm 2.65$ & $2.16 \pm 1.85$ & $1.78 \pm 0.92$ \\
\hline & control fish & $2.21 \pm 1.17$ & $5.93 \pm 5.79$ & $1.45 \pm 0.72$ & $2.51 \pm 2.65$ \\
\hline \multirow{2}{*}{$\mathrm{Ca}\left(\mathrm{mmol} \cdot \mathrm{l}^{-1}\right)$} & exposed fish & $4.29 \pm 1.06$ & $5.30 \pm 1.43$ & $5.61 \pm 1.57$ & $4.63 \pm 0.82$ \\
\hline & control fish & $5.50 \pm 1.20$ & $5.49 \pm 0.91$ & $5.26 \pm 0.99$ & $4.80 \pm 1.61$ \\
\hline \multirow{2}{*}{$\mathrm{TP}\left(\mathrm{g} \cdot \mathrm{l}^{-1}\right)$} & exposed fish & $37.9 \pm 3.7$ & $37.0 \pm 5.6$ & $36.7 \pm 5.3$ & $32.7 \pm 2.4^{*}$ \\
\hline & control fish & $37.6 \pm 3.4$ & $38.2 \pm 1.9$ & $35.1 \pm 4.4$ & $37.2 \pm 3.5$ \\
\hline \multirow{2}{*}{$\operatorname{GLC}\left(\mathrm{mmol} \cdot 1^{-1}\right)$} & exposed fish & $3.45 \pm 1.51$ & $4.25 \pm 1.60$ & $4.43 \pm 1.03$ & $3.10 \pm 0.50$ \\
\hline & control fish & $3.85 \pm 1.21$ & $4.14 \pm 2.11$ & $3.50 \pm 1.25$ & $3.24 \pm 1.08$ \\
\hline \multirow{2}{*}{$\operatorname{Mg}\left(\mathrm{mmol}^{-1} \mathrm{l}^{-1}\right)$} & exposed fish & $1.18 \pm 0.23$ & $1.17 \pm 0.14$ & $1.12 \pm 0.19$ & $1.01 \pm 0.07$ \\
\hline & control fish & $1.08 \pm 0.15$ & $1.14 \pm 0.18$ & $1.11 \pm 0.20$ & $1.16 \pm 0.07$ \\
\hline \multirow{2}{*}{$\mathrm{LACT}\left(\mathrm{mmol} \cdot \mathrm{l}^{-1}\right)$} & exposed fish & $0.88 \pm 0.62 *$ & $0.76 \pm 0.41$ & $1.21 \pm 0.54$ & $1.00 \pm 0.51$ \\
\hline & control fish & $0.28 \pm 0.11$ & $0.77 \pm 0.30$ & $0.91 \pm 0.51$ & $0.81 \pm 0.43$ \\
\hline \multirow{2}{*}{$\mathrm{LDH}\left(\mu \mathrm{kat} \cdot \cdot^{-1}\right)$} & exposed fish & $17.0 \pm 14.8$ & $17.1 \pm 12.1$ & $9.3 \pm 4.9$ & $8.6 \pm 4.9$ \\
\hline & control fish & $10.4 \pm 5.9$ & $29.8 \pm 29.5$ & $7.8 \pm 4.4$ & $9.0 \pm 10.9$ \\
\hline \multirow{2}{*}{$\mathrm{P}\left(\mathrm{mmol} \cdot \mathrm{l}^{-1}\right)$} & exposed fish & $2.00 \pm 0.50$ & $2.77 \pm 0.32$ & $3.03 \pm 1.15$ & $2.06 \pm 0.28 * *$ \\
\hline & control fish & $2.10 \pm 0.59$ & $2.71 \pm 0.69$ & $2.33 \pm 0.53$ & $3.84 \pm 0.67$ \\
\hline \multirow{2}{*}{$\mathrm{Fe}\left(\mu \mathrm{mol} \cdot 1^{-1}\right)$} & exposed fish & $17.3 \pm 3.0^{*}$ & $13.4 \pm 3.5$ & $6.2 \pm 2.6$ & $6.9 \pm 4.5$ \\
\hline & control fish & $12.6 \pm 4.7$ & $12.0 \pm 4.8$ & $6.9 \pm 4.4$ & $10.8 \pm 5.0$ \\
\hline \multirow{2}{*}{$\begin{array}{l}\mathrm{U} \\
\left(\mathrm{mol} \cdot \mathrm{1}^{-1}\right)\end{array}$} & exposed fish & $0.36 \pm 0.26^{* *}$ & $0.72 \pm 0.43$ & $0.24 \pm 0.15$ & $0.44 \pm 0.18$ \\
\hline & control fish & $0.85 \pm 0.25$ & $0.39 \pm 0.24$ & $0.22 \pm 0.13$ & $0.47 \pm 0.13$ \\
\hline \multirow{2}{*}{$\mathrm{CHE}\left(\mu \mathrm{kat} \cdot \cdot^{-1}\right)$} & exposed fish & $20.48 \pm 5.60$ & $3.9 \pm 1.45^{*}$ & $3.89 \pm 1.54$ & $4.10 \pm 1.51$ \\
\hline & control fish & $23.57 \pm 8.32$ & $20.56 \pm 15.32$ & $3.77 \pm 0.94$ & $3.88 \pm 1.63$ \\
\hline \multirow{2}{*}{$\mathrm{CHOL}\left(\mathrm{mmol} \cdot \mathrm{l}^{-1}\right)$} & exposed fish & $5.59 \pm 1.32$ & $6.86 \pm 2.43$ & $5.75 \pm 1.54$ & $5.81 \pm 1.88$ \\
\hline & control fish & $7.42 \pm 1.95$ & $7.49 \pm 1.26$ & $5.34 \pm 1.15$ & $6.41 \pm 1.45$ \\
\hline
\end{tabular}

Abbreviations: acid phosphatase (ACP), albumin (ALB), alkaline phosphatase (ALP), alanine aminotransferase (ALT), total bilirubin (BIL), creatinine (CRE), aspartate aminotransferase (AST), calcium (Ca), total protein (TP), glucose (GLC), magnesium (Mg), lactate (LACT), lactate dehydrogenase (LDH), phosphorus (P), iron (Fe), urea $(\mathrm{U})$, cholinesterase (CHE), cholesterol (CHOL), ${ }^{*}(p<0.05),{ }^{* *}(p<0.01)$.

cyanobacteria damaged parenchymatous tissues or skeletal musculature. Increased value of creatinine is an indicator of kidney damage, muscular dystrophia and physical exertion of the organism (Masopust 1998).

In previous experiments, values of total protein significantly decreased after intraperitoneal application of pure microcystin-LR into the common carp (Navratil et al. 1998), silver carp (Vajcova et al. 1998) or were not changed in the carp (Carbis et al. 1996). Changes of total protein under the influence of cyanobacterial populations were reduced in the common carp (Kopp and Hetesa 2000) and were not changed in the silver carp (Kopp 
et al. 2005). Values of total protein concentration in our experiment were not significantly different in comparison with the control group except for values on day 56 showing a significant decrease, whereas values of urea were increased on day 14 and then decreased on day 35 , probably due to modulation of metabolism in association with toxic stress.

Most authors have reported an increase in plasma lactate concentration in various fish following stress and the acute effects of toxic substances including bacterial toxins (Dabrowska et al. 1991; Kakuta et al. 1991; Williams et al. 1997; Kopp et al. 2005). In our experiment, a significant increase of lactate concentration (day 35) in blood plasma of experimental fish compared to the control may indicate a lower metabolic rate of lactate in the hepatopancreas and/or may be the result of stress from the effect of the cyanobacteria. The decrease of cholesterol levels may indicate slight damage of the hepatopancreas, similar to the significant increase of lactate levels. The negative effect of different pollutants at sublethal concentrations and stress of fish may be indicated by a decrease in cholesterol values (Gluth and Hanke 1985; Svobodova et al. 2006). However, no decrease of cholesterol was found in our study; on the contrary, we noticed an increase.

Our results showed significant differences in electrolytes $(\mathrm{Fe}, \mathrm{Mg}$ and $\mathrm{P})$ after exposure of the fish to cyanobacteria. The Mg value decreased at first (day 14) and then increased (day 21). Values of Fe and P increased (day 35) and decreased (day 56), respectively. There are reports on a significant decrease in values of $\mathrm{Ca}$ and $\mathrm{Mg}$, and a significant increase in $\mathrm{Fe}$ and $\mathrm{P}$ in the silver carp under the influence of a natural cyanobacterial population (Kopp et al. 2005). The basic function of electrolytes in the body lies in controlling fluid distribution, intra- and extracellular acid-base balance, maintaining osmotic pressure of body fluids and normal neuromuscular irritability. Calcium and phosphorus ions functionally participate in maintaining normal irritability of the heart, muscles and nerves, as well as the selective permeability of cell membranes. Magnesium and iron are important for normal function of the kidneys, liver and proteosynthesis. Decreased or increased values of electrolytes in blood plasma indicated abnormal function of fish organisms.

Biochemical indices in fish are affected by many endogenous and exogenous factors. Liver enzymes (ALT, AST and LDH) are the variables most suitable as indicators of the toxicity of cyanobacteria after intraperitoneal or oral biomass application in fish. The toxic effect of cyanobacteria in fish under natural environmental conditions is many times weaker than after the intraperitoneal or oral administration. Long action of the low concentration of MCs may cause only mild changes of liver parenchyma without significant increases in ALT, AST and LDH. Our results document the fact that liver damage was not so severe as to cause changes in the activities of liver enzymes. Other indices such as ALP and total bilirubin may be the sign of impaired bile secretion due to lower food intake as well as a decrease of total protein and an increase of lactate, which may also be associated with higher stress. In comparison to previous studies we may conclude that the Nile tilapia, which forages on algae filtered from the water using tiny combs in the gills, is less susceptible to cyanobacterial toxins than non-phytoplanktonophagous fish such as the common carp. There are two possible hypotheses concerning the difference in susceptibility of the Nile tilapia and common carp. First, cyprinid fish have longer intestines with greater absorption capacity and are thus able to accummulate higher toxin concentrations. Second, fish such as the Nile tilapia digesting cyanobacteria come into greater contact with toxins and thus have better and more effective detoxification mechanisms.

\section{Vybrané hematologické a biochemické ukazatele tilapie nilské (Oreochromis niloticus) chované v prostředí s vodním květem sinic}

Cílem studie bylo vyhodnotit vliv př́tomnosti toxického vodního květu sinic na krevní parametry tilapie nilské Oreochromis niloticus). Pokusné ryby byly vystaveny přirodnímu 
vodnímu květu sinic (sestávajícímu zejména z Microcystis aeruginosa a $M$. ichthyoblabe), jenž obsahoval microcystiny (celková koncentrace $1211 \mu \mathrm{g} \cdot \mathrm{g}^{-1}$ sušiny a $17.4-25.4 \mu \mathrm{g} \cdot \mathrm{l}^{-1}$ vody) po dobu 28 dní bez přikrmování. Kontrolní skupina ryb byla držena v nádrži bez zjevné tvorby cyanobakteriálního květu. Zdroj vody v pokusných i kontrolních nádržích byl stejný. Po 28 dnech byly ryby drženy po stejnou dobu v dechlorované pitné vodě.

Statistickým vyhodnocením jsme prokázali výrazný vzestup alkalické fosfatázy $(p \leq 0,05)$, celkového bilirubinu $(p \leq 0,001)$, kreatininu $(p \leq 0,01)$, laktátu $(p \leq 0,01)$ a močoviny $(p \leq 0,01)$ u experimentální skupiny ve srovnání s kontrolními rybami. Po přelovení ryb do pitné vody vykazovala experimentální skupina signifikantní snížení fosforu $(p \leq 0,001)$, močoviny $(p \leq 0,01)$ a cholinesterázy $(p \leq 0,05)$ a zvýšení hodnot laktázy $(p \leq 0,05)$ a železa $(p \leq 0,05)$ ve srovnání s kontrolní skupinou. Celkově lze říci, že pobyt tilapie nilské v prostředí obsahujícím toxický vodní květ sinic ovlivnila pouze některé biochemické ukazatele. Avšak tato modulace je na mnohem nižším stupni ve srovnání s kaprem obecným a tolstolobikem bílým.

\section{Acknowledgements}

This study was supported by the Ministry of Education, Youth and Sports of the Czech Republic "Veterinary aspects of food safety and quality" (MSM 62 15712402) and by the National Agency for Agricultural Research (QH 71015). Help from the Centre for Cyanobacteria and Their Toxins with analyses of microcystins is appreciated.

\section{References}

APHA 1981: Standard methods for the examination of water and wastewater (15 th edition) American Public Health Association Inc., Washington D.C.

Atencio L, Moreno I, Jos A, Prieto AI, Moryano R, Blanco A, Camean AM 2009: Effects of dietary selenium on the oxidative stress and pathological changes in tilapia (Oreochromis niloticus) exposed to a microcystinproducing cyanobacterial water bloom. Toxicon 53: 269-282

Blaha L, Marsalek B 2003: Contamination of drinking water in the Czech Republic by microcystins. Arch Hydrobiol 158: 421-429

Carbis CR, Mitchell GF, Anderson JW, McCauley I 1996: The effects of microcystins on the serum biochemistry of carp, Cyprinus carpio L., when the toxins are administered by gavage, immersion and intraperitoneal routes. J Fish Dis 19: 151-159

Carbis CR, Rawlin GT, Grant P, Mitchell GF, Anderson JW, McCauley I 1997: A study of feral carp, Cyprinus carpio L., exposed to Microcystis aeruginosa at Lake Mokoan, Australia, and possible implications for fish health. J Fish Dis 20: 81-91

Dabrowska H, Dabrowski K, Meyer-Burgdorff K, Hanke W, Gunther KD 1991: The effect of large doses of vitamin $\mathrm{C}$ and magnesium on stress responses in common carp Cyprinus carpio. Comp Biochem Phys A 99: $681-685$

Dobsikova R, Svobodova Z, Blahova J, Modra H, Velisek J 2006: Stress response to long distance transportation of common carp (Cyprinus carpio L.). Acta Vet Brno 75: 437-448

Gluth G, Hanke W 1985: A comparison of physiological changes in carp, Cyprinus carpio, induced by several pollutants at sublethal concentrations. Ecotox Environ Safe 9: 179-188

ISO 10260 1992: Water quality - Measurement of biochemical parameters - Spectrometric determination of the chlorophyll- $a$ concentration, Int. Org. Standard. Geneva $1^{\text {st }}$ edition 1992, 6 pp

Jos A, Pichardo S, Prieto AI, Repetto G, Vazquez CM, Moreno I, Camean AM 2005: Toxic cyanobacterial cells containing microcystins induce oxidative stress in exposed tilapia fish (Oreochromis sp.) under laboratory conditions. Aquat Toxicol 72: 261-271

Kakuta I, Namba K, Uematsu K, Murachi S 1991: Physiological response of the fish, Cyprinus carpio, to formalin exposure - I. Effects of formalin on urine flow, heart rate, respiration. Comp Biochem Phys C 100: 405-411

Kopp R, Hetesa J 2000: Changes of haematological indices of juvenile carp (Cyprinus carpio L.) under the influence of natural populations of cyanobacterial water blooms. Acta Vet Brno 69: 131-137

Kopp R, Mares J, Kubicek Z, Babica P 2005: The influence of toxic cyanobacterial water blooms on the hematological indicators of silver carp (Hypophthalmichthys molitrix Val.). Ocean Hydrobiol Stud 34: 85-92

Li XY, Chung IK, Kim JI, Lee JA 2004: Subchronic oral toxicity of microcystin in common carp (Cyprinus carpio L.) exposed to Microcystis under laboratory conditions. Toxicon 44: 821-827

Luskova V, Svoboda M, Kolarova J 2002: The Effect of Diazinon on Blood Plasma Biochemistry on Carp (Cyprinus carpio L.). Acta Vet Brno 71: 117-123

Malbrouck Ch, Kestemont P 2006: Effects of microcystins on fish. Environ Toxicol Chem 25: 72-86

Marsalek B, Blaha L, Turanek J, Neca J 2001: Microcystin-LR and total microcystins in cyanobacterial blooms in the Czech Republic 1993-2000. Cyanotoxins - Occurence, Causes, Consequences. I. Chorus. Berlin, Germany, Springer-Verlag: pp. 56-62 
Masopust J 1998: Clinical biochemistry. Demanding and evaluation of biochemical investigation. Karolinum Praha, 832 pp (in Czech)

Molina R, Moreno I, Pichardo S, Jos A, Moyano R, Monterde JG, Camean AM 2005: Acid and alkaline phosphatase activities and pathological changes induced in Tilapia fish (Oreochromis sp.) exposed subchronically to microcystins from toxic cyanobacterial blooms under laboratory conditions. Toxicon 46: 725-735

Navratil S, Palikova M, Vajcova V 1998: The effect of pure microcystin LR and biomass of blue-green algae on blood indices of carp (Cyprinus carpio L.). Acta Vet Brno 67: 273-279

Pepeljnjak S, Petrinec Z, Kovacic S, Segvic M 2003: Screening toxicity study in young carp (Cyprinus carpio L.) on feed amended with fumonisin B-1. Mycopathologia 156: 139-145

Prieto AI, Jos A, Pichardo S, Moreno I, Camean AM 2006: Differential oxidative stress responses to microcystins LR and RR in intraperitoneally exposed tilapia fish (Oreochromis sp.). Aquat Toxicol 77: 314-321

Prieto AI, Jos A, Pichardo S, Moreno I, Camean AM 2008: Protective role of vitamin E on the microcystininduced oxidative stress in tilapia fish (Oreochromis niloticus). Env Tox Chem 27(5): 1152-1159

Prieto AI, Pichardo S, Jos A, Moreno I, Camean AM 2007: Time-dependent oxidative stress responses after acute exposure to toxic cyanobacterial cells containing microcystins in tilapia fish (Oreochromis niloticus) under laboratory conditions. Aquat Toxicol 84: 337-345

Rabergh CMI, Bylund G, Eriksson JE 1991: Histopathological effect of microcystin LR a cyclic polypeptide from the cyanobacterium Microcystis aeruginosa on common carp (Cyprinus carpio L.). Aquat Toxicol 20: $131-146$

Ross C, Santiago-Vazquez L, Paul V 2006: Toxin release in response to oxidative stress and programmed cell death in the cyanobacterium Microcystis aeruginosa. Aquat Toxicol 78: 66-73

Serpunin G 1995: The connection between carp haematological indicators and its growth rate and its well-fedness. In: Proceedings of the $4^{\text {th }}$ Ichtyohaematological Conference, Hluboká nad Vltavou, Czech Republic: 21-22

Shen Q, Hu J, Li DH, Wang GH, Liu YD 2005: Investigation on intake, accumulation and toxicity of microcystins to silver carp. Fresenius Envir Bull 14: 1124-1128

Svobodova Z, Pravda D, Palackova J 1991: Unified methods of haematological examination of fish. Research Institute of Fish Culture and Hydrobiology, Vodňany, Edition Methods 22: $31 \mathrm{pp}$

Svobodova Z, Vykusova B, Modra H, Jarkovsky J, Smutna M 2006: Haematological and biochemical profile of harvest-size carp during harvest and post-harvest storage. Aquac Res 37: 959-965

Tencalla GF, Dietrich RD, Schlatter Ch 1994: Toxicity of Microcystis aeruginosa peptide toxin to yearling rainbow trout (Oncorhynchus mykiss). Aquat Toxicol 30: 215-224

Vajcova V, Navratil S, Palikova M 1998: The effect of intraperitoneally applied pure microcystin LR on haematological, biochemical and morphological indices of silver carp (Hypophthalmichthys molitrix Val.). Acta Vet Brno 67: 281-287

Williams EM, Nelson JA, Heisler N 1997: Cardio-respiratory function in carp exposed to environmental nitrite. J Fish Biol 50: 137-149 